\title{
THE CURVATURE OF HALF LIGHTLIKE SUBMANIFOLDS OF A SEMI-RIEMANNIAN MANIFOLD OF QUASI-CONSTANT CURVATURE
}

\author{
DAE Ho JiN
}

\begin{abstract}
We study half lightlike submanifolds $M$ of semi-Riemannian manifolds $\widetilde{M}$ of quasi-constant curvatures. The main result is a characterization theorem for screen homothetic Einstein half lightlike submanifolds of a Lorentzian manifold of quasi-constant curvature subject to the conditions; (1) the curvature vector field of $\widetilde{M}$ is tangent to $M$, and (2) the co-screen distribution is a conformal Killing one.
\end{abstract}

\section{INTRODUCTION}

Chen and Yano [1] introduced the notion of a Riemannian manifold of quasiconstant curvature as a Riemannian manifold $(\widetilde{M}, \widetilde{g})$ equipped with the curvature tensor $\widetilde{R}$ satisfying the following condition:

$$
\begin{aligned}
\widetilde{g}(\widetilde{R}(X, Y) Z, W)= & \alpha\{\widetilde{g}(Y, Z) \widetilde{g}(X, W)-\widetilde{g}(X, Z) \widetilde{g}(Y, W)\} \\
& +\beta\{\widetilde{g}(X, W) \theta(Y) \theta(Z)-\widetilde{g}(X, Z) \theta(Y) \theta(W) \\
& +\widetilde{g}(Y, Z) \theta(X) \theta(W)-\widetilde{g}(Y, W) \theta(X) \theta(Z)\},
\end{aligned}
$$

where $\alpha, \beta$ are scalar functions and $\theta$ is a 1 -form defined by

$$
\theta(X)=\widetilde{g}(X, \zeta)
$$

and $\zeta$ is a unit vector field on $\widetilde{M}$, which called the curvature vector field of $\widetilde{M}$. It is well known that if the curvature tensor $\widetilde{R}$ is of the form (1.1), then $\widetilde{M}$ is conformally flat. If $\beta=0$, then $\widetilde{M}$ is a space of constant curvature $\alpha$.

Recently Jin [7] and Jin and Lee [8] studied lightlike submanifolds $M$ in a semiRiemannian manifold $\widetilde{M}$ of quasi-constant curvature subject to the conditions; (1)

Received by the editors June 7, 2012. Accepted October 25, 2012.

2010 Mathematics Subject Classification. 53C25, 53C40, 53C50.

Key words and phrases. screen homothetic, conformal Killing distribution, half lightlike submanifold, semi-Riemannian manifold of quasi-constant curvature. 
the curvature vector field $\zeta$ of $\widetilde{M}$ is tangent to $M$ and (2) the screen distribution is totally geodesic in $M$. They proved two characterization theorems for such lightlike submanifolds (see $[7,8]$ ).

The classification of Einstein half lightlike submanifolds $M$ was studied by Jin [5]. Its main result focused on the geometry of Einstein half lightlike submanifolds $M$ of a Lorentz space form $\widetilde{M}(c)$ of constant curvature $c$, whose co-screen distribution is a Killing one and whose shape operator is conformal to the shape operator of its screen distribution by some non-vanishing smooth function $\varphi$. The reason for this geometric restrictions on $M$ was due to the fact that such a class admits an integrable screen distribution and a symmetric induced Ricci tensor. After that, Jin [6] generalized the main result of [5] for Einstein screen conformal half lightlike submanifold of Lorentz space forms endow with a conformal Killing co-screen distribution. A careful proof of [6] is even more involved than that of [5]. He proved a characterization theorem for such half lightlike submanifolds as it follow:

Theorem 1.1. Let $M$ be a screen conformal half lightlike submanifold of a Lorentz space form $\widetilde{M}^{m+3}(c)(m>2)$ of constant curvature $c$ equipped with a conformal Killing co-screen distribution of conformal factor $\delta$. If $M$ is Einstein, i.e., Ric $=\kappa g$, then $M$ is locally a product manifold $\mathcal{C} \times M_{1} \times M_{2}$, where $\mathcal{C}$ is a null curve and $M_{1}$ and $M_{2}$ are totally umbilical leaves of some distributions of $M$ :

(1) If $\kappa \neq(m-1)\left(c+\delta^{2}\right)$, then either $M_{1}$ or $M_{2}$ is an m-dimensional Einstein Riemannian space form which is isometric to a sphere $(\kappa>0)$ or a hyperbolic space $(\kappa<0)$ and the other is a point on $M$.

(2) If $\kappa=(m-1)\left(c+\delta^{2}\right)$, then $M_{1}$ is an $(m-1)$ or $m$-dimensional Einstein Riemannian space form which is isometric to a sphere $(\kappa>0)$ or a hyperbolic space $(\kappa<0)$ or a Euclidean space $(\kappa=0)$ and $M_{2}$ is a spacelike curve or a point on $M$.

In particular, if the co-screen distribution is a Killing one, then $c=\delta=0$ in the conditional paragraph of above two cases (1) and (2).

The objective of this paper is to generalize the above characterization theorem for screen homothetic Einstein half lightlike submanifolds of a Lorentzian manifold of quasi-constant curvature. We prove a characterization theorem for screen homothetic half lightlike submanifolds $M$ of a Lorentzian manifold $\widetilde{M}$ of quasi-constant curvature subject to the condition; (1) the curvature vector field of $\widetilde{M}$ is tangent to $M$, and (2) the co-screen distribution is a conformal Killing one. 


\section{Half Lightlike Submanifolds}

It is well known that the radical distribution $\operatorname{Rad}(T M)=T M \cap T M^{\perp}$ of half lightlike submanifolds $M$ of a semi-Rimannian manifold $(\widetilde{M}, \widetilde{g})$ of codimension 2 is a vector subbundle of the tangent bundle $T M$ and the normal bundle $T M^{\perp}$, of rank 1 . Therefore there exist complementary non-degenerate distributions $S(T M)$ and $S\left(T M^{\perp}\right)$ of $\operatorname{Rad}(T M)$ in $T M$ and $T M^{\perp}$ respectively, which called the screen distribution and co-screen distribution on $M$, such that

$$
T M=\operatorname{Rad}(T M) \oplus_{\text {orth }} S(T M), \quad T M^{\perp}=\operatorname{Rad}(T M) \oplus_{\text {orth }} S\left(T M^{\perp}\right),
$$

where $\oplus_{\text {orth }}$ denotes the orthogonal direct sum. We denote such a half lightlike submanifold by $M=\left(M, g, S(T M), S\left(T M^{\perp}\right)\right)$. Denote by $F(M)$ the algebra of smooth functions on $M$ and by $\Gamma(E)$ the $F(M)$ module of smooth sections of any vector bundle $E$ over $M$. Consider the orthogonal complementary distribution $S(T M)^{\perp}$ to $S(T M)$ in $T \widetilde{M}$. Certainly $T M^{\perp}$ is a subbundle of $S(T M)^{\perp}$. As $S\left(T M^{\perp}\right)$ is a non-degenerate subbundle of $S(T M)^{\perp}$, the orthogonal complementary distribution $S\left(T M^{\perp}\right)^{\perp}$ of $S\left(T M^{\perp}\right)$ in $S(T M)^{\perp}$ is also a non-degenerate distribution such that

$$
S(T M)^{\perp}=S\left(T M^{\perp}\right) \oplus_{\text {orth }} S\left(T M^{\perp}\right)^{\perp} .
$$

Clearly $\operatorname{Rad}(T M)$ is a vector subbundle of $S\left(T M^{\perp}\right)^{\perp}$. Choose $L \in \Gamma\left(S\left(T M^{\perp}\right)\right)$ as a unit vector field with $\widetilde{g}(L, L)=\epsilon= \pm 1$. For any null section $\xi$ of $\operatorname{Rad}(T M)$, there exists a uniquely defined null vector field $N \in \Gamma\left(S\left(T M^{\perp}\right)^{\perp}\right)$ satisfying

$$
\widetilde{g}(\xi, N)=1, \widetilde{g}(N, N)=\widetilde{g}(N, X)=\widetilde{g}(N, L)=0, \forall X \in \Gamma(S(T M))
$$

Denote by $\operatorname{lt}(T M)$ the subbundle of $S\left(T M^{\perp}\right)^{\perp}$ locally spanned by $N$. Then we show that $S\left(T M^{\perp}\right)^{\perp}=\operatorname{Rad}(T M) \oplus \operatorname{ltr}(T M)$. Let $\operatorname{tr}(T M)=S\left(T M^{\perp}\right) \oplus_{\text {orth }} l \operatorname{tr}(T M)$. We call $N$, $\operatorname{tr}(T M)$ and $\operatorname{tr}(T M)$ the lightlike transversal vector field, lightlike transversal vector bundle and transversal vector bundle of $M$ with respect to the screen distribution $S(T M)$ respectively [3]. Then $T \widetilde{M}$ is decomposed as follow:

$$
\begin{aligned}
T \widetilde{M} & =T M \oplus \operatorname{tr}(T M)=\{\operatorname{Rad}(T M) \oplus \operatorname{tr}(T M)\} \oplus_{\text {orth }} S(T M) \\
& =\{\operatorname{Rad}(T M) \oplus \operatorname{ltr}(T M)\} \oplus_{\text {orth }} S(T M) \oplus_{\text {orth }} S\left(T M^{\perp}\right) .
\end{aligned}
$$

Let $\widetilde{\nabla}$ be the Levi-Civita connection of $\widetilde{M}$ and $P$ the projection morphism of $T M$ on $S(T M)$ with respect to the decomposition (2.1). Then the local Gauss and 
Weingarten formulas of $M$ and $S(T M)$ are given by

$$
\begin{aligned}
& \widetilde{\nabla}_{X} Y=\nabla_{X} Y+B(X, Y) N+D(X, Y) L, \\
& \widetilde{\nabla}_{X} N=-A_{N} X+\tau(X) N+\rho(X) L, \\
& \widetilde{\nabla}_{X} L=-A_{L} X+\phi(X) N \\
& \nabla_{X} P Y=\nabla_{X}^{*} P Y+C(X, P Y) \xi \\
& \nabla_{X} \xi=-A_{\xi}^{*} X-\tau(X) \xi, \quad \forall X, Y \in \Gamma(T M),
\end{aligned}
$$

where $\nabla$ and $\nabla^{*}$ are induced connections on $T M$ and $S(T M)$ respectively, $B$ and $D$ are called the local second fundamental forms of $M, C$ is called the local second fundamental form on $S(T M) . A_{N}, A_{\xi}^{*}$ and $A_{L}$ are linear operators on $T M$ and $\tau, \rho$ and $\phi$ are 1-forms on $T M$. Since $\widetilde{\nabla}$ is torsion-free, the induced connection $\nabla$ of $M$ is also torsion-free and both $B$ and $D$ are symmetric. From the facts $B(X, Y)=\widetilde{g}\left(\widetilde{\nabla}_{X} Y, \xi\right)$ and $D(X, Y)=\epsilon \widetilde{g}\left(\widetilde{\nabla}_{X} Y, L\right)$, we know that $B$ and $D$ are independent of the choice of a screen distribution and

$$
B(X, \xi)=0, \quad D(X, \xi)=-\epsilon \phi(X) .
$$

The induced connection $\nabla$ on $M$ is not metric and satisfies

$$
\left(\nabla_{X} g\right)(Y, Z)=B(X, Y) \eta(Z)+B(X, Z) \eta(Y)
$$

where $\eta$ is a 1 -form on $T M$ such that $\eta(X)=\widetilde{g}(X, N)$. But the connection $\nabla^{*}$ on $M^{*}$ is metric. The above three local second fundamental forms of $M$ and $M^{*}$ are related to their shape operators by

$$
\begin{array}{ll}
B(X, Y)=g\left(A_{\xi}^{*} X, Y\right), & \widetilde{g}\left(A_{\xi}^{*} X, N\right)=0, \\
C(X, P Y)=g\left(A_{N} X, P Y\right), & \widetilde{g}\left(A_{N} X, N\right)=0, \\
\epsilon D(X, Y)=g\left(A_{L} X, Y\right)-\phi(X) \eta(Y), & \widetilde{g}\left(A_{L} X, N\right)=\epsilon \rho(X) .
\end{array}
$$

By (2.10) and (2.11), we show that $A_{\xi}^{*}$ and $A_{N}$ are $\Gamma(S(T M))$-valued shape operators related to $B$ and $C$ respectively and $A_{\xi}^{*}$ is self-adjoint on $T M$ and

$$
A_{\xi}^{*} \xi=0
$$

Denote by $\widetilde{R}, R$ and $R^{*}$ the curvature tensors of the Levi-Civita connection $\widetilde{\nabla}$ on $\widetilde{M}$, the induced connection $\nabla$ on $M$ and the induced connection $\nabla^{*}$ on $S(T M)$ respectively. Using the Gauss-Weingarten equations for $M$ and $S(T M)$, for any 
$X, Y, Z \in \Gamma(T M)$, we obtain the following Codazzi equations for $M$ and $S(T M)$ :

$$
\begin{gathered}
\widetilde{g}(\widetilde{R}(X, Y) Z, \xi)=\left(\nabla_{X} B\right)(Y, Z)-\left(\nabla_{Y} B\right)(X, Z) \\
+B(Y, Z) \tau(X)-B(X, Z) \tau(Y) \\
+D(Y, Z) \phi(X)-D(X, Z) \phi(Y), \\
\widetilde{g}(\widetilde{R}(X, Y) Z, N)=\widetilde{g}(R(X, Y) Z, N) \\
+\epsilon\{D(X, Z) \rho(Y)-D(Y, Z) \rho(X)\}, \\
\widetilde{g}(\widetilde{R}(X, Y) \xi, N)=g\left(A_{\xi}^{*} X, A_{N} Y\right)-g\left(A_{\xi}^{*} Y, A_{N} X\right) \\
\quad-2 d \tau(X, Y)+\rho(X) \phi(Y)-\rho(Y) \phi(X), \\
\widetilde{g}(R(X, Y) P Z, N)=\left(\nabla_{X} C\right)(Y, P Z)-\left(\nabla_{Y} C\right)(X, P Z) \\
+C(X, P Z) \tau(Y)-C(Y, P Z) \tau(X) .
\end{gathered}
$$

The Ricci curvature tensor, denoted by $\widetilde{R i c}$, of $\widetilde{M}$ is defined by

$$
\widetilde{\operatorname{Ric}}(X, Y)=\operatorname{trace}\{Z \rightarrow \widetilde{R}(Z, X) Y\}
$$

for any $X, Y \in \Gamma(T \widetilde{M})$. Let $\operatorname{dim} \widetilde{M}=m+3$. Locally, $\widetilde{R i c}$ is given by

$$
\widetilde{\operatorname{Ric}}(X, Y)=\sum_{i=1}^{m+3} \epsilon_{i} \widetilde{g}\left(\widetilde{R}\left(E_{i}, X\right) Y, E_{i}\right),
$$

where $\left\{E_{1}, \ldots, E_{m+3}\right\}$ is an orthonormal frame field of $T \widetilde{M}$ and $\epsilon_{i}(= \pm 1)$ denotes the causal character of respective vector field $E_{i}$. Consider a quasi-orthonormal frame field $\left\{\xi ; W_{a}\right\}$ on $M$ such that $\operatorname{Rad}(T M)=\operatorname{Span}\{\xi\}$ and $S(T M)=\operatorname{Span}\left\{W_{a}\right\}$, and let $E=\left\{\xi, W_{a}, N, L\right\}$ be the corresponding frame field on $\widetilde{M}$. Using this frame field, for all $X, Y \in \Gamma(T M)$, the equation (2.18) reduce to

$$
\begin{aligned}
\widetilde{\operatorname{Ric}}(X, Y) & =\sum_{a=1}^{m} \epsilon_{a} \widetilde{g}\left(\widetilde{R}\left(W_{a}, X\right) Y, W_{a}\right)+\widetilde{g}(\widetilde{R}(\xi, X) Y, N) \\
& +\epsilon \widetilde{g}(\widetilde{R}(L, X) Y, L)+\widetilde{g}(\widetilde{R}(N, X) Y, \xi) .
\end{aligned}
$$

Definition. A vector field $X$ on a semi-Riemannian manifold $(\bar{M}, \bar{g})$ is said to be a conformal Killing vector field $[5,6]$ if $\overline{\mathcal{L}}_{X} \bar{g}=-2 \delta \bar{g}$ for any non-vanishing smooth function $\delta$, where $\overline{\mathcal{L}}_{X}$ denotes the Lie derivative with respect to $X$, that is,

$$
\left(\overline{\mathcal{L}}_{X} \bar{g}\right)(Y, Z)=X(\bar{g}(Y, Z))-\bar{g}([X, Y], Z)-\bar{g}(Y,[X, Z]), \forall Y, Z \in \Gamma(T \bar{M}) .
$$

In particular, if $\delta=0$, then $X$ is called a Killing vector field [5]. A distribution $\mathcal{G}$ on $\bar{M}$ is called a conformal Killing (resp. Killing) distribution on $\bar{M}$ if each vector field belonging to $\mathcal{G}$ is a conformal Killing (resp. Killing) vector field on $\bar{M}$. 
Theorem 2.1 ([5, 6]). Let $M$ be a half lightlike submanifold of a semi-Riemannian manifold $(\widetilde{M}, \widetilde{g})$. Then $S\left(T M^{\perp}\right)$ is a conformal Killing distribution if and only if there exists a smooth function $\delta$ such that

$$
D(X, Y)=\epsilon \delta g(X, Y), \quad \forall X, Y \in \Gamma(T M) .
$$

Proof. By using (2.5) and (2.12), for any $X, Y \in \Gamma(T M)$, we have

$$
\begin{gathered}
\left(\widetilde{\mathcal{L}}_{L} \widetilde{g}\right)(X, Y)=\widetilde{g}\left(\widetilde{\nabla}_{X} L, Y\right)+\widetilde{g}\left(X, \widetilde{\nabla}_{Y} L\right), \\
\widetilde{g}\left(\widetilde{\nabla}_{X} L, Y\right)=-g\left(A_{L} X, Y\right)+\phi(X) \eta(Y)=-\epsilon D(X, Y) .
\end{gathered}
$$

From $\left(\widetilde{\mathcal{L}}_{L} \widetilde{g}\right)(X, Y)=-2 \epsilon D(X, Y)$ we deduce our assertion.

\section{MAIn THEOREM}

Let $M$ be a half lightlike submanifold of a semi-Riemannian manifold $\widetilde{M}$ of quasiconstant curvature. Assume that the curvature vector field $\zeta$ of $\widetilde{M}$ is a unit spacelike vector field of $M$. If $\zeta$ belongs to $\operatorname{Rad}(T M)$, then $\zeta=e \xi$, where $e=\theta(N) \neq 0$. From this fact, we have $1=\widetilde{g}(\zeta, \zeta)=e^{2} g(\xi, \xi)=0$. It is a contradiction. This enables one to choose a screen distribution $S(T M)$ which contains $\zeta$. This implies that if $\zeta$ is tangent to $M$, then it belongs to $S(T M)$ which we assume in this paper.

Definition. A half lightlike submanifold $M$ of a semi-Riemannian manifold $\widetilde{M}$ is screen conformal $[4,5,6]$ if the shape operators $A_{N}$ and $A_{\xi}^{*}$ of $M$ and $S(T M)$ respectively are related by $A_{N}=\varphi A_{\xi}^{*}$, or equivalently, the second fundamental forms $B$ and $C$ of $M$ and $S(T M)$ respectively satisfy

$$
C(X, P Y)=\varphi B(X, Y),
$$

where $\varphi$ is a non-vanishing smooth function on a coordinate neighborhood $\mathcal{U}$ in $M$. If $\varphi$ is a non-zero constant, then we say that $M$ is screen homothetic.

Theorem 3.1. Let $M$ be a screen conformal half lightlike submanifold of a semiRiemannian manifold $(\widetilde{M}, \widetilde{g})$ of quasi-constant curvature. If $\zeta$ is tangent to $M$ and $\phi=0$, then the 1-form $\tau$ is closed, i.e., $d \tau=0$, on $T M$.

Proof. Replacing $W$ by $N$ to (1.1) and using the fact $\theta(N)=0$, we have

$$
\begin{aligned}
\widetilde{g}(\widetilde{R}(X, Y) Z, N) & =\alpha\{\eta(X) g(Y, Z)-\eta(Y) g(X, Z)\} \\
& +\beta\{\theta(Y) \eta(X)-\theta(X) \eta(Y)\} \theta(Z) .
\end{aligned}
$$

Replacing $Z$ by $\xi$ to (3.2) and using $\theta(\xi)=0$, we have $\widetilde{g}(\widetilde{R}(X, Y) \xi, N)=0$. 
Comparing this result with $(2.16)$ and using the facts $A_{N}=\varphi A_{\xi}^{*}$ and $\phi=0$, we show that the 1 -form $\tau$ is closed, i.e., $d \tau=0$, on $T M$.

Note 1. In case $d \tau=0$, by the cohomology theory there exist a smooth function $l$ such that $\tau=d l$. Thus we get $\tau(X)=X(l)$. If we take $\tilde{\xi}=\gamma \xi$, then we have $\tau(X)=\tilde{\tau}(X)+X(\ln \gamma)$. Setting $\gamma=\exp (l)$ in this equation, we get $\tilde{\tau}(X)=0$. We call the pair $\{\xi, N\}$ such that the corresponding 1-form $\tau$ vanishes the canonical null pair of $M$. Although $S(T M)$ is not unique but it is canonically isomorphic to the factor vector bundle $S(T M)^{\sharp}=T M / \operatorname{Rad}(T M)$ due to Kupeli [9]. Thus all $S(T M)$ are mutually isomorphic. In the sequel, we deal with only half lightlike submanifolds $M$ equipped with the canonical null pair.

Theorem 3.2. Let $M$ be a screen homothetic half lightlike submanifold of a semiRiemannian manifold $\widetilde{M}$ of quasi-constant curvature such that the curvature vector field $\zeta$ of $\widetilde{M}$ is tangent to $M$.

(1) If $S\left(T M^{\perp}\right)$ is Killing, then the functions $\alpha$ and $\beta$, given by (1.1), vanish identically, and $\widetilde{M}$ is a flat manifold.

(2) If $S\left(T M^{\perp}\right)$ is conformal Killing, then the functions $\beta$, given by (1.1), vanishes identically, and $\widetilde{M}$ is a space of constant curvature $\alpha$.

Proof. Using (1.1), (2.18) and the facts $\theta(\xi)=\theta(N)=\theta(L)=0$, we have

$$
\begin{aligned}
& \widetilde{R i c}(X, Y)=\{(m+2) \alpha+\beta\} g(X, Y)+(m+1) \beta \theta(X) \theta(Y), \\
& \widetilde{g}(\widetilde{R}(\xi, Y) X, N)=\alpha g(X, Y)+\beta \theta(X) \theta(Y), \\
& \epsilon \widetilde{g}(\widetilde{R}(L, Y) X, L)=\alpha g(X, Y)+\beta \theta(X) \theta(Y), \quad \forall X, Y \in \Gamma(T M) .
\end{aligned}
$$

As $S\left(T M^{\perp}\right)$ is conformal Killing, from (2.8), (2.12) and (2.20) we have

$$
D(X, Y)=\epsilon \delta g(X, Y), \quad \phi=0, \quad A_{L} X=\delta P X+\epsilon \rho(X) \xi .
$$

As $d \tau=0$ by Theorem 3.1, we can take a canonical null pair such that $\tau=0$ by Note 1. Replacing $W$ by $\xi$ to (1.1) and using (2.14) and the fact $\theta(\xi)=0$, we have

$$
\left(\nabla_{X} B\right)(Y, Z)-\left(\nabla_{Y} B\right)(X, Z)=0, \quad \forall X, Y, Z \in \Gamma(T M) .
$$

As $M$ is screen homothetic, substituting (3.1) into (2.17) and using (3.7), we get $\widetilde{g}(R(X, Y) P Z, N)=0$. From this, $(2.15)$ and the fact $\widetilde{g}(\widetilde{R}(X, Y) \xi, N)=0$, we have

$$
\widetilde{g}(\widetilde{R}(X, Y) Z, N)=\delta\{g(X, Z) \rho(Y)-g(Y, Z) \rho(X)\} .
$$

Replacing $X$ by $\xi$ and $Z$ by $X$ to this and comparing with (3.4), we have

$$
\beta \theta(X) \theta(Y)=-\{\alpha+\delta \rho(\xi)\} g(X, Y), \quad \forall X, Y \in \Gamma(T M) .
$$


Taking $X=Y=\zeta$ to (3.8), we get $\beta=-\{\alpha+\delta \rho(\xi)\}$. Substituting (3.8) into (3.3) and using the fact $\beta=-\{\alpha+\delta \rho(\xi)\}$, we obtain

$$
\widetilde{\operatorname{Ric}}(X, Y)=-(m+2) \delta \rho(\xi) g(X, Y) \quad \forall X, Y \in \Gamma(T M) .
$$

Substituting (3.8) into (1.1) and using the fact $\beta=-\{\alpha+\delta \rho(\xi)\}$, we have

$$
\bar{g}(\widetilde{R}(X, Y) Z, W)=(\alpha+2 \delta \rho(\xi))\{g(X, Z) g(Y, W)-g(Y, Z) g(X, W)\},
$$

for all $X, Y, Z, W \in \Gamma(T M)$. Substituting (3.4), (3.5), (3.10) into (2.19), we have

$$
\widetilde{\operatorname{Ric}}(X, Y)=-\{(m-1) \alpha+(2 m+1) \delta \rho(\xi)\} g(X, Y) .
$$

Comparing (3.9) and (3.11), we have $\alpha+\delta \rho(\xi)=0$ as $m>1$. Thus we have $\beta=0$.

Case (1). If $S\left(T M^{\perp}\right)$ is Killing distribution, then $\delta=0$. In this case, we get $\alpha=\beta$. As $\beta=0$, we obtain $\alpha=\beta=0$. Therefore $\widetilde{M}$ is a flat manifold.

Case (2). If $S\left(T M^{\perp}\right)$ is conformal Killing distribution, then $\delta \neq 0$. In this case, we get $\alpha=-\delta \rho(\xi)$ and $\beta=0$. Therefore $\widetilde{M}$ is a space of constant curvature $\alpha$.

By Theorem 1.1, we have the following characterization theorem:

Theorem 3.3. Let $M$ be a screen homothetic half lightlike submanifold of a Lorentz manifold $\widetilde{M}^{m+3}(m>2)$ of quasi-constant curvature. If the curvature vector field $\zeta$ of $\widetilde{M}$ is tangent to $M$, the co-screen distribution $S\left(T M^{\perp}\right)$ is conformal Killing of conformal factor $\delta$ and $M$ is Einstein, i.e., Ric $=\kappa g$, then $M$ is locally a product manifold $\mathcal{C} \times M_{1} \times M_{2}$, where $\mathcal{C}$ is a null curve tangent to the radical distribution, and $M_{1}$ and $M_{2}$ are totally umbilical leaves of some distributions of $M$ :

(1) If $\kappa \neq(m-1)\left(\alpha+\delta^{2}\right)$, then either $M_{1}$ or $M_{2}$ is an $m$-dimensional Einstein Riemannian space form which is isometric to a sphere $(\kappa>0)$ or a hyperbolic space $(\kappa<0)$ and the other is a point on $M$.

(2) If $\kappa=(m-1)\left(\alpha+\delta^{2}\right)$, then $M_{1}$ is an $(m-1)$ or $m$-dimensional Einstein Riemannian space form which is isometric to a sphere $(\kappa>0)$ or a hyperbolic space $(\kappa<0)$ or a Euclidean space $(\kappa=0)$ and $M_{2}$ is a spacelike curve or a point on $M$.

Corollary 1. Let $M$ be a screen homothetic Einstein half lightlike submanifold of a Lorentzian manifold $\widetilde{M}, m>2$, of quasi-constant curvature equipped with a Killing co-screen distribution. Then $\widetilde{M}$ is a flat manifold, and $M$ is a locally product manifold $\mathcal{C} \times M_{1} \times M_{2}$, where $\mathcal{C}$ is a null curve, and $M_{1}$ and $M_{2}$ are leaves of some distributions of $M$ such that 
(1) If $\kappa \neq 0$, then either $M_{1}$ or $M_{2}$ is an m-dimensional Einstein Riemannian space form which is isometric to a sphere $(\kappa>0)$ or a hyperbolic space $(\kappa<$ $0)$ and the other is a point on $M$.

(2) If $\kappa=0, M_{1}$ is an $(m-1)$ or an $m$-dimensional Euclidean space and $M_{2}$ is a spacelike curve or a point in $\bar{M}$.

\section{REFERENCES}

1. B.Y. Chen \& K. Yano: Hypersurfaces of a conformally flat space. Tensor (N. S.) 26 (1972), 318-322.

2. K.L. Duggal \& A. Bejancu: Lightlike Submanifolds of Semi-Riemannian Manifolds and Applications. Kluwer Acad. Publishers, Dordrecht, 1996.

3. K.L. Duggal \& D.H. Jin: Half-lightlike submanifolds of codimension 2. Math. J. Toyama Univ. 22 (1999), 121-161.

4. __ : Null curves and Hypersurfaces of Semi-Riemannian Manifolds. World Scientific, 2007.

5. D.H. Jin: Einstein half lightlike submanifolds with a Killing co-screen distribution. Honam Math. J. 30 (2008), no. 3, 487-504.

6. E__ Einstein half lightlike submanifolds with special conformalities. accepted in Bull. Korean Math. Soc., 2012.

7. _ L L Lightlike hypersurfaces of a semi-Riemannian manifold of quasi-constant curvature. accepted in Commun. Korean Math. Soc., 2011.

8. D.H. Jin \& J.W. Lee: Lightlike submanifolds of a semi-Riemannian manifold of quasiconstant curvature. accepted in J. of Appl. Math., 2012.

9. D.N. Kupeli: Singular Semi-Riemannian Geometry. Mathematics and Its Applications, vol. 366, Kluwer Acad. Publishers, Dordrecht, 1996.

Department of Mathematics, Dongguk University, Gyeongju 780-714, Republic of KoREA

Email address: jindh@dongguk.ac.kr 